\title{
Trends in recent waterborne and foodborne disease outbreaks in South Korea, 2015-2019
}

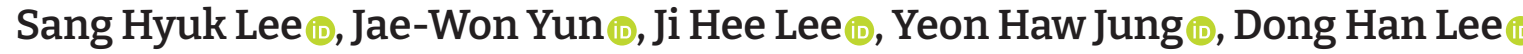 \\ Infectious Disease Control, Center for Infectious Disease Control, Korea Disease Control and Prevention Agency, Cheongju, Korea
}

Received: September 24, 2020

Revised: February 16, 2021

Accepted: February 24, 2021

Corresponding author:

Dong Han Lee

Infectious Disease Control,

Center for Infectious Disease

Control, Korea Disease Control

and Prevention Agency, 187

Osongsaengmyeong 2-ro,

Osong-eup, Heungdeok-gu,

Cheongju 28159, Korea

E-mail: Idhmd@korea.kr

\section{ABSTRACT}

Objectives: This study analyzed trends in foodborne and waterborne diseases in South Korea between 2015 and 2019.

Methods: The data consisted of information on outbreaks of waterborne and foodborne infectious diseases reported through the Korea Centers for Disease Control and Prevention (KCDC) system. We analyzed the trends and epidemiological aspects of outbreaks by month, place of occurrence, and causative pathogens in this observational study.

Results: The number of outbreaks has steadily increased over the last 5 years, but the number of cases per outbreak has followed a decreasing trend. Incidence at daycare centers and preschools has been steadily increasing over consecutive years.

Conclusion: The steady number of patients and decreasing number of cases per outbreak, even as the number of outbreaks has been increasing, suggest that the KCDC's professional management system is operating effectively. It is necessary to continue improving the objectivity and efficiency of the management system and to carefully examine the increasing number of outbreaks in smaller-scale group catering facilities, such as daycare centers and preschools. Outbreaks can be prevented by closely examining those caused by unidentified pathogens and group outbreaks caused by other diseases, identifying problems, and supplementing the management system.

Keywords: Foodborne diseases; Korea; Outbreaks; Waterborne disease

\section{Introduction}

Since 2007, the Korea Centers for Disease Control and Prevention (KCDC) has managed outbreaks of waterborne and foodborne infectious diseases that occur in South Korea (hereafter, Korea), while also collecting the results of epidemiological investigations. The World Health Organization defines a waterborne or foodborne disease outbreak as "a case in which two or more people experienced the same disease after eating the same food or drinking water from the same source" [1]. In Korea, the occurrence of waterborne and foodborne infectious diseases is defined as the appearance of gastroenteritis symptoms, such 
as diarrhea or vomiting, in a group of 2 or more people, with epidemiological associations (e.g., time and place) [2]. A status analysis provides basic, critical data that may be used to prepare policies for the prevention of infectious diseases. Unlike previous studies, this study subdivided outbreaks according to their place of occurrence and analyzed their timing and the causative pathogens. The purpose of this study was to analyze the status of outbreaks of waterborne and foodborne infectious diseases that occurred in Korea from 2015 to 2019 and to examine trends in those outbreaks.

\section{Materials and Methods}

This study analyzed outbreaks of waterborne and foodborne infectious diseases reported through the KCDC's web reporting system from 2015 to 2019. Reports on the results of all epidemiological investigations must be submitted to the KCDC within 14 days after the end of the outbreak, and the reports submitted are reviewed by a KCDC Epidemic Intelligence Service officer, evaluated, and managed as data.

Outbreaks were excluded from the analysis if they were not reported in the results of an epidemiological investigation or if an epidemiological connection had not yet been confirmed. We analyzed the trends and epidemiological aspects of outbreaks by month, place of occurrence, and causative pathogens. Microsoft Excel was used for the analysis. The study protocol was approved by the KCDC Institutional Review Board (IRB No: 2020-08-03$\mathrm{PE}-\mathrm{A}$ ). Informed consent was confirmed by the IRB.

\section{Results}

From 2015 to 2019, a total of 2,815 waterborne and foodborne disease outbreaks involving 48,032 cases were reported to the KCDC (Figure 1). There was an increase from 2015 to 2018, but the number of outbreaks decreased in 2019. However, the number of cases per outbreak followed a decreasing trend. In 2018 , there was an increase of $29.3 \%$ in the number of outbreaks ( $n=697)$ compared to the previous year $(n=539)$, and the number of cases approximately doubled compared to the previous year. With the exception of a peak in 2018, the number of outbreaks has been steadily increasing over the last 5 years.

Outbreaks occurred most intensively between May and September (9.5\%, 9.2\%, 9.3\%, 9.8\%, and 10.2\%, respectively) (Table 1), accounting for $48 \%$ of the total annual outbreaks. However, May and June 2015 accounted for a lower percentage of outbreaks (6.9\%) than the corresponding months in other years (Figure 2). Outbreaks most commonly occurred in March and June in elementary, middle, and high schools and least commonly in January and February. July and August, which are the summer vacation period, accounted for $16.4 \%$ of outbreaks among elementary, middle, and high school groups, likely due to active afterschool learning activities. The highest incidence in daycare centers occurred in winter (November, December, and January) and the lowest occurred in summer (July and August). Similarly, the incidence of outbreaks in preschools was highest in winter, while it was relatively low in summer. For restaurants, the highest incidence was during summer,

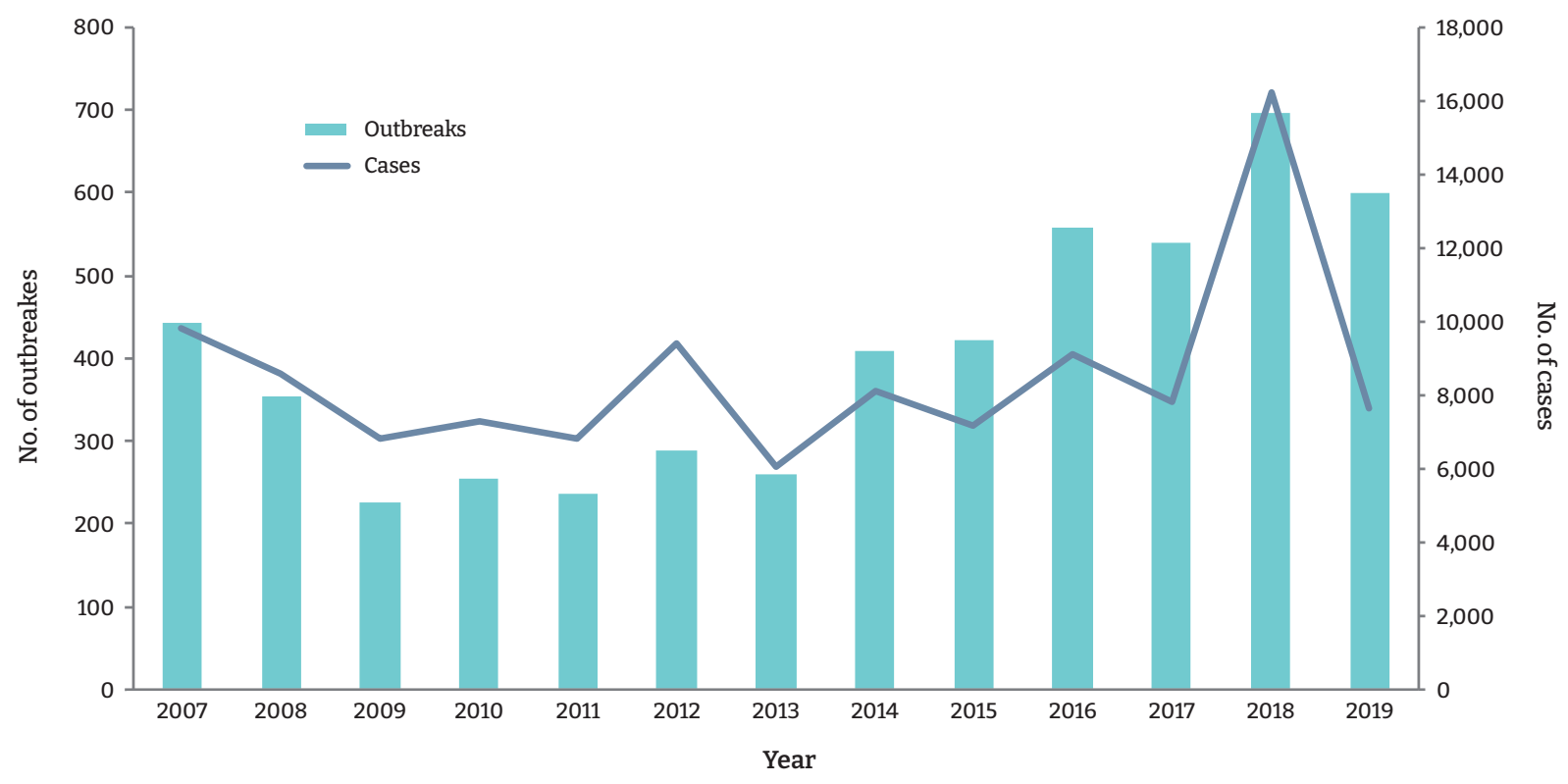

Figure 1. Number of reported waterborne and foodborne disease outbreaks and cases. 
Table 1. Number of reported waterborne and foodborne disease outbreaks and cases by month

\begin{tabular}{|c|c|c|c|c|c|c|c|c|c|c|c|c|}
\hline \multirow{2}{*}{ Month } & \multicolumn{2}{|c|}{2015} & \multicolumn{2}{|c|}{2016} & \multicolumn{2}{|c|}{2017} & \multicolumn{2}{|c|}{2018} & \multicolumn{2}{|c|}{2019} & \multicolumn{2}{|c|}{ Total (\%) } \\
\hline & Outbreak & Case & Outbreak & Case & Outbreak & Case & Outbreak & Case & Outbreak & Case & Outbreak & Case \\
\hline January & 52 & 537 & 22 & 192 & 38 & 296 & 28 & 178 & 48 & 503 & $188(6.7)$ & 1,706 (3.6) \\
\hline February & 17 & 217 & 17 & 89 & 33 & 215 & 37 & 557 & 35 & 275 & 139 (4.9) & $1,353(2.8)$ \\
\hline March & 35 & 414 & 33 & 452 & 33 & 351 & 61 & 1,334 & 56 & 988 & $218(7.7)$ & $3,539(7.4)$ \\
\hline April & 33 & 490 & 49 & 567 & 50 & 858 & 47 & 848 & 67 & 1,012 & $246(8.7)$ & 3,775 (7.9) \\
\hline May & 29 & 500 & 53 & 871 & 51 & 691 & 62 & 1,213 & 72 & 873 & $267(9.5)$ & $4,148(8.6)$ \\
\hline June & 29 & 732 & 48 & 766 & 65 & 904 & 51 & 1,068 & 66 & 952 & $259(9.2)$ & $4,422(9.2)$ \\
\hline July & 45 & 612 & 34 & 459 & 56 & 753 & 69 & 979 & 58 & 772 & $262(9.3)$ & $3,575(7.4)$ \\
\hline August & 42 & 2,212 & 72 & 2,516 & 65 & 1,346 & 50 & 1,586 & 48 & 517 & 277 (9.8) & $8,177(17.0)$ \\
\hline September & 41 & 477 & 53 & 576 & 50 & 1,179 & 96 & 5,762 & 48 & 315 & $288(10.2)$ & 8,309 (17.3) \\
\hline October & 38 & 308 & 56 & 795 & 25 & 379 & 79 & 970 & 29 & 344 & $227(8.1)$ & $2,796(5.8)$ \\
\hline November & 32 & 276 & 57 & 929 & 30 & 369 & 62 & 880 & 35 & 577 & $216(7.7)$ & $3,031(6.3)$ \\
\hline December & 29 & 400 & 63 & 927 & 43 & 471 & 55 & 872 & 38 & 531 & $228(8.1)$ & $3,201(6.7)$ \\
\hline Total & 422 & 7,175 & 557 & 9,139 & 539 & 7,812 & 697 & 16,247 & 600 & 7,659 & $2,815(100)$ & $48,032(100)$ \\
\hline
\end{tabular}

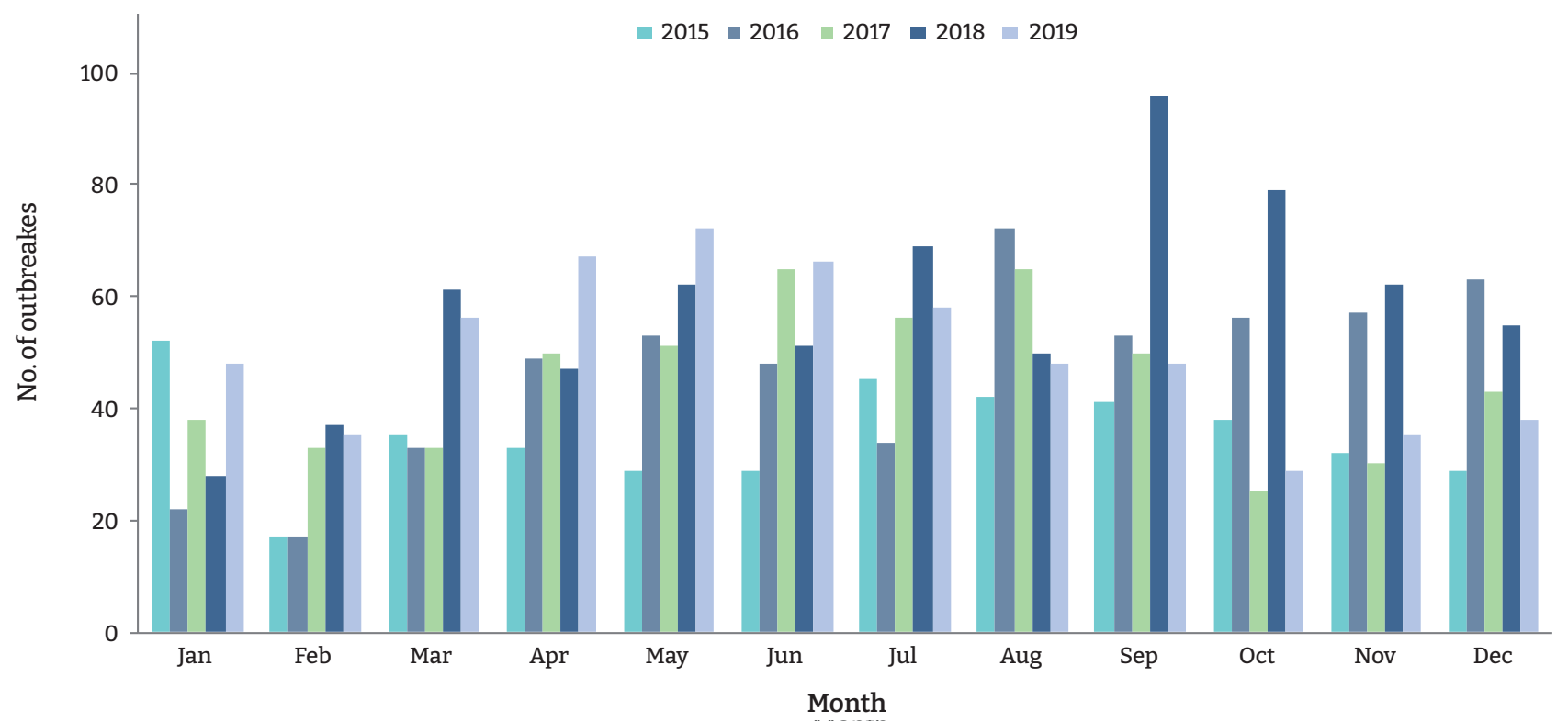

Figure 2. Number of reported waterborne and foodborne disease outbreaks by month.

between June and August (data not shown).

The causative pathogens were only identified in $58.7 \%$ of cases, of which norovirus was the most frequent, at $21.6 \%$ (Table 2). Aside from norovirus, the category of "others" accounted for $11.9 \%$, pathogenic Escherichia coli for 6.3\%, and Salmonella spp. for 3.5\%. "Others" included pathogens that are not legally defined as communicable diseases, such as Kudoa septempunctata and intestinal coliform infection. Two or more pathogens were involved in 3.2\% of outbreaks between 2015 and 2019. The majority (80.4\%) of outbreaks involving bacterial pathogens, such as pathogenic $E$. coli, occurred from May to October. Norovirus infections occurred most commonly in winter (January and December), accounting for $33.5 \%$ of the yearly incidence, with $22.7 \%$ of norovirus cases occurring from May to October.

Of the total norovirus outbreaks, $37.8 \%$ occurred in elementary, middle, and high schools, and $25.6 \%$ occurred in daycare centers and preschools. That is, $63.4 \%$ of norovirus outbreaks occurred in group facilities with minors. Norovirus displayed a yearly increasing trend, which was especially evident in 2018 , with an increase of $77.2 \%$ compared to the previous year.

The causative pathogens classified as "others" also increased annually due to an increase in the yearly number of outbreaks 


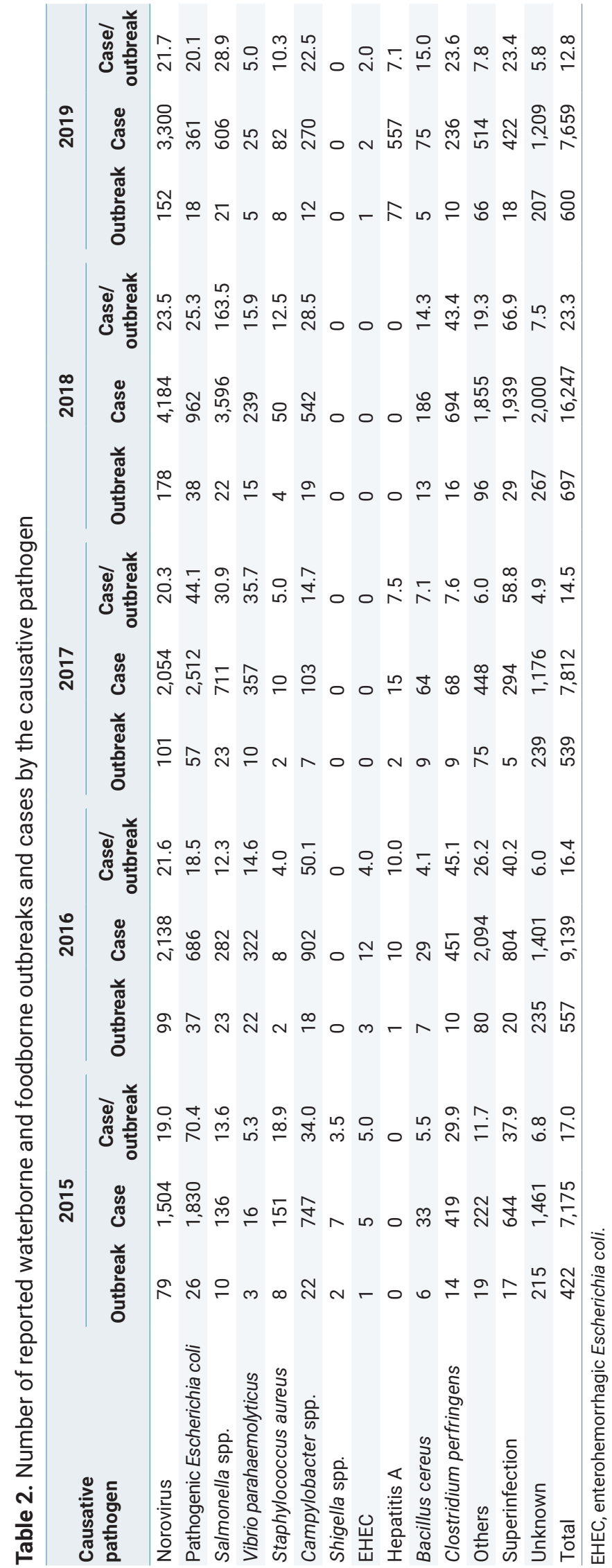

caused by $K$. septempunctata. In addition, there were 77 outbreaks related to hepatitis A in 2019, accounting for $3.7 \%$ of the total outbreaks that year. Notably, hepatitis A was previously not prominent in outbreaks. The proportion of outbreaks for which the causative pathogens could not be identified was $41.3 \%$, corresponding to approximately 200 outbreaks every year. The proportion of outbreaks for which the causative pathogen was classified as "unknown" decreased gradually over time (2015, 50.9\%; 2016, 42.2\%; 2017, 44.3\%; 2018, 38.3\%; 2019, 39.5\%). The places with the highest proportion of "unknown" pathogens were homes (60.3\%) and restaurants (56.1\%), and those with the fewest were preschools (5.4\%) and daycare centers (9.3\%).

General restaurants accounted for the highest proportion of outbreaks (48.0\%), although this category accounted for only $18.0 \%$ of total cases (Table 3 ). Conversely, the proportion of outbreaks in elementary, middle, and high schools was low (18.6\%), but the number of cases was approximately $50 \%$, or roughly half of all cases. The proportion of outbreaks occurring in daycare centers and preschools was $7.2 \%$, and the proportion of cases was $6.6 \%$. The proportion of outbreaks with unidentifiable locations was $5.4 \%$, and the proportion of cases was $2.4 \%$. Outbreaks in elementary, middle, and high schools and in restaurants showed increasing and decreasing trends similar to those of outbreaks overall, but the proportion of outbreaks at daycare centers and preschools steadily increased (2015, 4.0\%; 2016, 6.3\%; 2017, 5.4\%; 2018, 7.9\%; 2019, 11\%) (Table 4). Conversely, the proportion of outbreaks occurring at home decreased over the study period (2015, 19.4\%; 2016, 4.5\%; 2017, 2.0\%; 2018, 2.4\%; 2019, 4.8\%).

\section{Discussion}

The incidence of waterborne and foodborne infectious diseases in Korea exhibited an increasing trend over the past 5 years. From 2007 to 2009, the incidence of outbreaks gradually decreased, and the number of patients increased, prompting the suggestion that professional management of outbreaks was required [3]. In contrast, the findings of the present study show that despite the increase in the number of outbreaks in recent years, the number of cases has been relatively consistent every year, and the number of cases per outbreak has been decreasing. This can be interpreted as reflecting a lower incidence of outbreaks in group facilities, such as major catering facilities, which often have a major effect when they occur. These results also suggest that the professional management system is operating effectively. Since 2003, the KCDC has been monitoring the outbreak of waterborne and foodborne infectious diseases 


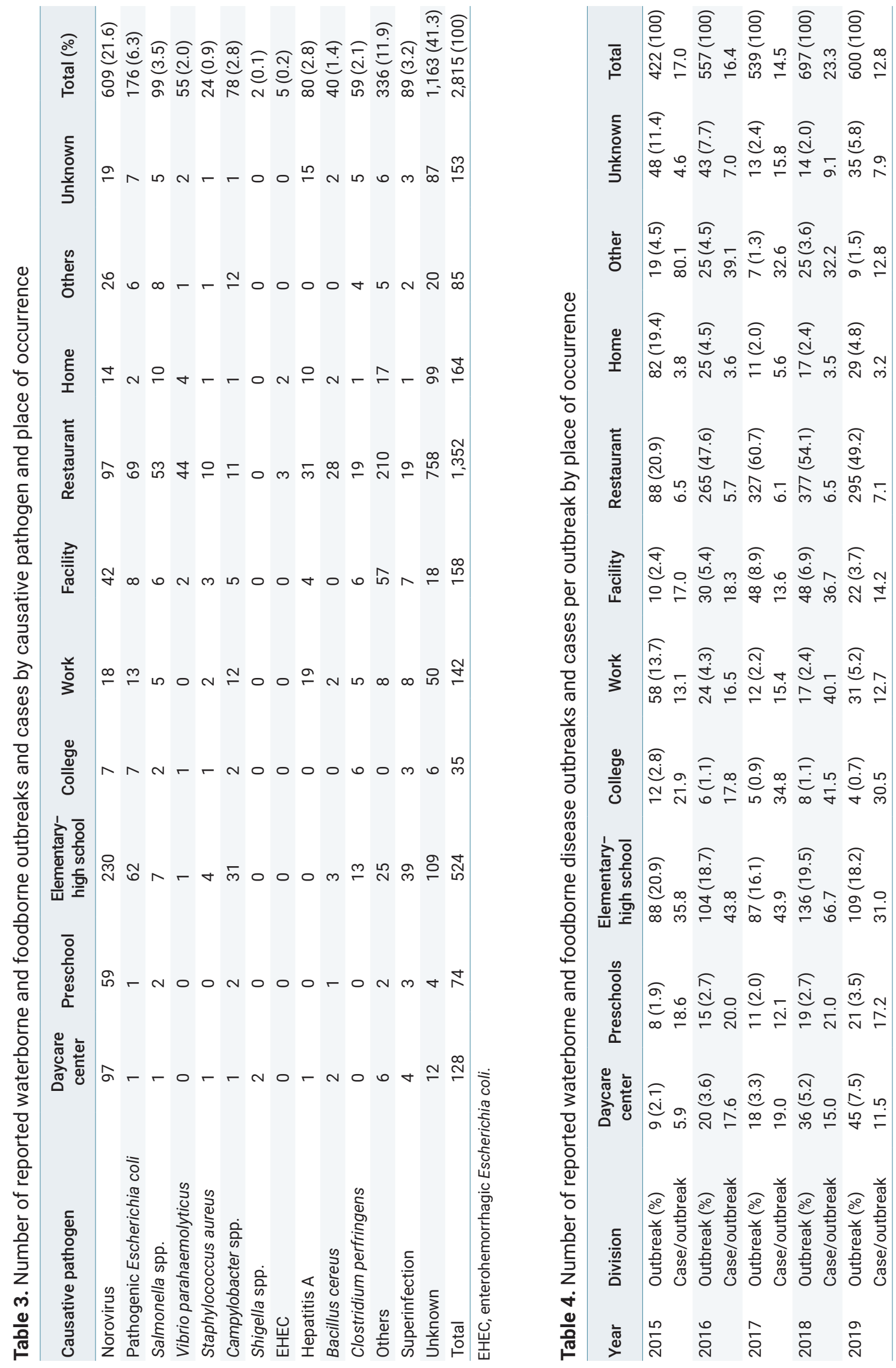


with real-time information through the Enteric Pathogens Active Surveillance Network (Enter-Net). Since 2012, efforts have also been made to prevent collective outbreaks of waterborne and foodborne infectious disease by expanding the monitoring of bacterial pathogens from 5 to 10 species [4].

Approximately 100 outbreaks occurred each year from 1995 to 2005, but the number of outbreaks increased to 259 in 2006 and 510 in 2007. The reason for these outbreaks was an increase in the use of group catering services, and the peak in 2007, in particular, occurred due to a change in the criteria used to define outbreaks from 5 to 2 persons affected [3]. However, approximately 540 outbreaks occurred recently, and the numbers are increasing over time. This may be caused by an increase in the culture of dining out and food delivery, the increasing use of group catering facilities, and improvements in the competence of health staff and the reporting system.

Approximately half of the outbreaks occurred between May and September each year. However, May and June of 2015 showed a lower proportion (6.9\%) than the corresponding months in the other years included in this study. This was likely caused by the outbreak of Middle East respiratory syndrome in Korea in 2015, which reduced activities outside the home and increased personal hygiene awareness, resulting in a decrease in the number of outbreaks occurring in restaurants and catering facilities. The KCDC conducts intensive monitoring from May to September every year to prevent outbreaks in the summer. This is because the high temperature and highly humid environment facilitate the proliferation of bacteria or viruses that cause outbreaks. Normally, outbreaks occurring from May to September account for approximately 57\% of the yearly total [3], but in recent years, outbreaks occurring from April to September have accounted for approximately the same proportion. This could be related to climate change causing the weather to be relatively warm in April. A $1^{\circ} \mathrm{C}$ rise in temperature increases the incidence of foodborne outbreaks by approximately 5\% [5]. According to data from the Korea Meteorological Administration, the average temperature in April from 2015 to 2019 was higher than the average temperature in April from 2007 to 2009 (2007, $11.7^{\circ} \mathrm{C} ; 2008,13.0^{\circ} \mathrm{C} ; 2009,12.6^{\circ} \mathrm{C} ; 2015,12.7^{\circ} \mathrm{C} ; 2016$, $13.8^{\circ} \mathrm{C} ; 2017,13.9^{\circ} \mathrm{C} ; 2018,13.3^{\circ} \mathrm{C} ; 2019,12.0^{\circ} \mathrm{C}$ ). This indicates that there is a correlation between the rise in temperature and outbreaks of waterborne and foodborne infectious diseases.

Norovirus is the most common pathogen that causes waterborne and foodborne infectious diseases. Furthermore, $16.5 \%$ of the outbreaks that occurred between 2007 and 2009 were caused by norovirus, $13.9 \%$ were caused by intestinal infections, and 39.9\% were caused by unidentified pathogens [3]. The number of outbreaks caused by norovirus has been higher in recent years than previously. Moreover, the proportion of the total outbreaks attributed to norovirus among those with identified pathogens was very high, at $36.9 \%$. The proportion of norovirus outbreaks worldwide is also high. For example, in the United States, norovirus accounted for approximately half (46\%) of all pathogen outbreaks between 2009 and 2015. More than half of the norovirus outbreaks occurred in group catering facilities related to minors and during summer. Due to the nature of norovirus, which is transmitted through contact, it occurs predominantly in groups involving minors. In addition, with the increase in after-school activities, norovirus, which was once mainly prevalent only in winter, is now showing a trend to spread in summer.

Outbreaks of pathogenic E. coli decreased by $6.3 \%$, but the number of cases for which the cause could not be identified rose to $41.3 \%$. The proportions of other causative pathogens, such as $K$. septempunctata, also significantly increased. In particular, outbreaks caused by K. septempunctata accounted for $80 \%$ of "other" pathogens and are increasing consistently on a yearly basis. Previously, outbreaks due to hepatitis A were uncommon in Korea. However, 17,598 cases occurred in Korea during 2019. Seventy-seven hepatitis A-related group outbreaks were reported, including 2 outbreaks with more than 100 cases each.

The proportion of outbreaks of Salmonella has significantly decreased. Salmonella outbreaks accounted for $12.9 \%$ of all outbreaks from 2003 to 2006 [6] and 7.7\% from 2007 to 2009 [3]. This decrease is due to the systemic management of Salmonella bacteria from the egg laying process to the egg distribution process by the Ministry of Agriculture, Food and Rural Affairs and the Ministry of Food and Drug Safety. However, cases associated with Salmonella outbreaks have increased steadily. In particular, in 2018, cases attributed to Salmonella outbreaks were approximately 5 times more numerous than in the previous year. The increase was attributed to the occurrence of Salmonella contamination in chocolate cakes, which are a popular product; this accounted for 2,975 cases in 2018.

Consistent with past trends, more than half of water and foodborne infectious disease outbreaks occurred in restaurants.

However, most of the group outbreaks that occurred in restaurants were small-scale outbreaks of 7 or fewer people, whereas large-scale outbreaks of more than 7 people frequently occurred in group catering facilities. In recent years, the frequency of outbreaks in group catering facilities, such as schools, has been increasing. The 
average proportion of outbreaks in schools was previously reported to be $14.5 \%$ [3], but the proportion of outbreaks occurring in elementary, middle, and high schools over the previous 5 years increased to $18.6 \%$. In particular, the proportion of outbreaks in daycare centers and preschools exhibited a steadily increasing trend every year from 2015 to 2019. However, according to statistics from the National Statistical Office, the number of children who attend daycare in Korea is gradually decreasing. That is, the number of children who use daycare centers is decreasing, but the number of outbreaks occurring in daycare centers is increasing. Therefore, it is necessary to strengthen the management system for daycare centers and preschools, which appear to be a blind spot with respect to waterborne and foodborne infectious diseases, and to decrease the incidence in these locations to the same level as in elementary, middle, and high schools.

In addition, the proportion of "unknown" cases, in which the causal pathogen was not identified, increased from 39.9\% (2007-2009) to 41.3\% (2015-2019). However, the number of patients per outbreak in "unknown" cases almost halved, from 11.1 (2007-2009) to 6.2 (2015-2019) [3]. "Unknown" cases were less frequent in preschools and daycare centers than in homes and restaurants. This indicates that places such as schools have better management systems for infectious diseases than do homes and restaurants. These results suggest that the professional management system is operating effectively.

\section{Conclusion}

The incidence of outbreaks of waterborne and foodborne infectious diseases in Korea has fluctuated in recent years, but showed an overall increase compared to the past. However, the present findings confirmed that the number of cases per outbreak decreased, while the number of overall patients remained relatively constant. These results indicate the positive effect of intensively managing largescale group catering facilities, such as schools, through professional human resources to prevent outbreaks. It is necessary to carefully examine the increasing number of outbreaks in smaller-scale group catering facilities, such as daycare centers and preschools. Group outbreaks among preschool children who cannot ensure their own personal hygiene must be systematically managed in collaboration with related ministries. In addition, outbreaks of waterborne and foodborne infectious diseases can be prevented by closely examining outbreaks due to unidentified pathogens and group outbreaks caused by other diseases; that is, steps should be taken to determine the cause of incidents, identify causative problems, and supplement the management system.

\section{Notes}

\section{Ethics Approval}

The study protocol was approved by the Institutional Review Board (IRB) of the Korea Centers for Disease Control and Prevention (IRB No: 202008-03-PE-A). Informed consent was confirmed by the IRB.

\section{Conflicts of Interest}

The authors have no conflicts of interest to declare.

\section{Funding}

None.

\section{Availability of Data}

All data generated or analysed during this study are included in this published article.

\section{Authors' Contributions}

Conceptualization: DHL; Data curation: SHL, JWY, JHL, YHJ; Formal analysis: SHL; Investigation: All author; Methodology: DHL, SHL; Project administration: JHL; Resources: SHL; Visualization: JHL; Writingoriginal draft: SHL, DHL; Writing-review \& editing: All authors.

\section{Additional Contributions}

We thank all the local government infectious disease managers.

\section{References}

1. World Health Organization (WHO). Foodborne disease outbreaks: guidelines for investigation and control. Geneva: WHO; 2008.

2. Korea Centers for Disease Control and Prevention (KCDC). Management guidelines for water $\&$ foodborne diseases [Internet]. Cheongju (KR): KCDC; 2020 [cited 2020 Sep 1]. Available from: https://www.kdca.go.kr/ upload_comm/syview/doc.html?fn =158209329137500.pdf\&rs =/ upload_comm/docu/0019/.

3. Gwack J, Lee KC, Lee HJ, et al. Trends in water- and foodborne disease outbreaks in Korea, 2007-2009. Osong Public Health Res Perspect 2010;1:50-4.

4. Kim NO, Hong S, Chun JH, et al. Laboratory-based surveillance of water- and food-borne infectious disease-causing bacteria in the Republic of Korea, 2016-2018. Public Health Wkly Rep 2019;12:898903. Korean.

5. Shin HS, Yun SM, Jung KH, et al. Climate change, food-borne disease prediction, and future impact. Health Soc Welf Rev 2009;29:143-62. Korean

6. Kwun JW, Lee CH. Trends of recent food-borne disease outbreaks in Korea. J Korean Med Assoc 2007;50:573-81. 